\title{
Tumor neuroendocrino Tipo 1: reporte de un caso y revisión de la literatura
}

\author{
Francisco Ortega G. ${ }^{1,2}$, Nicolás Yáñez B. ${ }^{1,2}$, César Muñoz C. ${ }^{1,2}$ y Dahiana Andrea Pulgar B. ${ }^{1,2}$
}

\section{Localized neuroendocrine tumor of the stomach. A case report and literature review}

Introduction: Neuroendocrine tumors (NETs) are composed of cells that produce peptides and amines Gastric NETs represent 1\% of all neoplasms; however their incidence has been increasing. They are usually asymptomatic and non-functioning. The treatment is usually local resection. Case Report: We present the case of a 48-year-old patient who was suspected of gastric cancer; her study shows a well-differentiated type 1 gastric NET. Staging is performed and endoscopic surveillance is defined in the oncology board. The finding of a NET, in the study of gastric cancer, is a rare finding. Due to the progressive increase in the performance of upper gastrointestinal endoscopies, secondary to the high prevalence of gastric cancer in our country, it is expected to increase findings as a NET. That is why we conducted a review of the literature and made some conclusions about it.

Key words: gastric endocrine tumours; gastric carcinoids; hypergastrinaemia; atrophic body gastritis; Zollinger-Ellison syndrome.

\section{Resumen}

Introducción: Los tumores neuroendocrinos (TNE), son tumores compuestos por células productoras de péptidos y aminas. Los TNE gástricos, representan el 1\% de todas las neoplasias, sin embargo su incidencia ha ido en aumento. Son generalmente asintomáticos y no funcionantes. El tratamiento es generalmente la resección local. Caso Clínico: paciente de 48 años con sospecha de cáncer gástrico; su estudio demuestra un TNE gástrico bien diferenciado tipo 1. Se realiza etapificación y se define en comité oncológico la vigilancia endoscópica. El hallazgo de un TNE, en el estudio de cáncer gástrico, es un hallazgo poco frecuente. Debido al aumento progresivo en la realización de endoscopías digestivas altas, secundario a la alta prevalencia de cáncer gástrico en nuestro país, se espera que aumenten hallazgos como un TNE. Es por esto que realizamos una revisión de la literatura y planteamos algunas conclusiones al respecto.

Palabras clave: tumor neuroendocrino gástrico; carcinoide gástrico; hipergastrinemia; gastritis atrófica; síndrome de Zollinger-Ellison.
'Hospital Regional de Talca. ¿Universidad Católica del Maule.

Recibido el 6 de junio de 2019 y aceptado para publicación el 5 de septiembre de 2019

Correspondencia a: Dra. Diana A. Pulgar B. dapulgar@gmail.com

\section{Introducción}

Los tumores neuroendocrinos (TNE), son tumores derivados del sistema neuroendocrino difuso, el cual se encuentra compuesto de células productoras de péptidos y aminas, que pueden producir diferentes hormonas dependiendo del sitio de origen ${ }^{1}$. Los TNE gástricos representan el $1 \%$ de todas las neoplasias y su incidencia se ha elevado en los últimos 50 años. En una serie de EE. UU., el porcentaje de estos tumores entre todas las neoplasias gástricas, ha aumentado de $0,3 \%$ a $1,8 \%$ y la proporción de estos TNE entre todos los carcinoides, de $2,4 \%$ a $8,7 \%{ }^{2}$. Si bien este aumento puede ser real, también puede ser explicado por el mayor uso de endoscopía y la evolución en el diagnóstico histopatológico. Así, en la era preendoscópica se estimaba que los TNE gástricos eran el 1,9\% de todos los carcinoides $\mathrm{y}$, actualmente, se estima que el $10 \%$ a $30 \%$ de todos los TNE son gástricos ${ }^{3}$. 


\section{Caso Clínico}

Paciente femenino de 48 años, sin antecedentes mórbidos de relevancia ni historia familiar conocida. Presenta historia de 4 meses de evolución de dolor epigástrico urente, no asociado a bochornos, diarrea, baja de peso u otros síntomas. Es derivado a través de sistema GES (garantías explícitas en salud), dado que en su diagnóstico diferencial se encuentra la sospecha de cáncer gástrico.

Se realiza endoscopía digestiva alta (EDA) que muestra gastropatía eritematosa y un pólipo de 4 $\mathrm{mm}$ en fondo gástrico que se extirpa con pinza. Estudio histológico muestra tumor neuroendocrino bien diferenciado grado 1 limitado a la mucosa. Mitosis $3 \mathrm{x} \mathrm{mm}^{2}$. La inmunohistoquímica muestra CD56 (+), cromogranina (+), sinaptofisina (+). Ki67 menos del $2 \%$.

Es presentada en comité oncológico, se decide evaluación por oncología médica y repetir EDA. Evaluada por equipo de oncología, se realiza TC de tórax, abdomen y pelvis que no muestra otras lesiones. Gastrina $347 \mathrm{pg} / \mathrm{mL}(\mathrm{VN}<90)$, cromogranina A: $159 \mathrm{ng} / \mathrm{mL}(\mathrm{VN}<108)$. No es posible realizar estudio funcional de etapificación, dado que no se encuentra disponible en nuestro sistema público de salud.

Se repite EDA, que muestra lesiones polipoides múltiples en el fondo gástrico (más de 6), todas menores de $10 \mathrm{~mm}$. Se realiza biopsia de una lesión. Biopsia informa, en la microscopía, lesión polipoide en parte conformada por células normotípicas y un foco de células monótonas dispuestas en nidos y cordones, con núcleos hipercromáticos, sin mitosis. Hallazgos compatibles con tumor neuroendocrino bien diferenciado. Inmunohistoquímica muestra Ki67 5\% CD56 (+) Sinaptofisina (+) Pancitokeratina $(+)$.

Es nuevamente evaluada en comité oncológico, donde se concluye como diagnóstico, tumor neuroendocrino gástrico bien diferenciado Tipo 1. Dado que es paciente asintomática, con lesiones incontables, y considerando preferencia de la misma, se decide seguimiento clínico y vigilancia endoscópica anual.

Evoluciona asintomática del punto de vista gastrointestinal. Destaca en su evolución, episodios aislados de rubicundez facial no compatibles con bochornos.

\section{Discusión}

Obendorfer y Frankfurt acuñaron el término carcinoide en 1907 para separar un grupo de tumores del intestino delgado que se comportaban menos agresivos que los carcinomas convencionales ${ }^{4}$. Tumores carcinoides gástricos, fueron reportados por primera vez por Askanazy en 19235. Actualmente, la Organización Mundial de la Salud, clasifica estos tumores como neuroendocrinos ${ }^{6}$, siendo el término carcinoide aplicado para tumores neuroendocrinos bien diferenciados originados en el tracto gastrointestinal, broncopulmonar u otros sitios infrecuentes.

La mucosa gástrica, se divide tanto funcional como histológicamente en 2 tipos; la que cubre la región antropilórica y el cuerpo-fondo. Ambas se caracterizan por tener poblaciones distintas de células endocrinas; la región antropilórica posee predominantemente células $\mathrm{G}$ secretoras de gastrina, y la región cuerpo-fondo células tipo enterocromafines (ECL) secretoras de histamina 7 . Así los TNE son extremadamente raros en el antro, y son predominantemente de células ECL.

La histamina, estimula la secreción ácida de células parietales adyacentes. Las células ECL expresan receptores CCK-2 (gastrina), y pueden mediar la secreción de histamina y estimular el crecimiento celular. Entonces, la hipergastrinemia crónica está asociada con hiperplasia de células ECL y con el tiempo puede derivar en un TNE de células $\mathrm{ECL}^{8}$.

Los TNE gástrico, pueden dividirse en 4 tipos $^{7,8}$ :

\section{Tipo 1}

Representa entre el $70 \%$ y el $80 \%$ de los TNE gástricos. Se asocia con gastritis atrófica; sin embargo, menos del $1 \%$ de los pacientes con gastritis atrófica presentan un TNE Tipo $1^{9}$. Su edad media de presentación es 51 años y es más frecuente en mujeres ${ }^{10}$. Del punto de vista morfológico, son habitualmente tumores pequeños, que parecen lesiones polipoideas. Pueden ser únicos o múltiples y tienen morfología típica de carcinoide. La hipotésis de patogénesis es que la célula ECL se transforma a TNE después de la estimulación crónica por altos niveles de gastrina que son consecuencia de la gastritis atrófica ${ }^{3}$. Estos tumores son habitualmente no funcionantes, se presentan asintomáticos, y por lo general representan una condición benigna ${ }^{9}$. Las metástasis son raras; ocurren en aproximadamente un $10 \%$ en tumores menores a $10 \mathrm{~mm}$ y en $30 \%$ en tumores mayores a $20 \mathrm{~mm}^{11}$.

\section{Tipo 2}

Ocurren en asociación con gastrinomas (síndrome de Zollinger-Ellison (SZE)), comúnmente en el contexto de una neoplasia endocrina múltiple (NEM 1). Representan aproximadamente el 5\%-10\% de 
los TNE gástricos. Los pacientes con SZE asociado a NEM1, tienen un riesgo de 20\%-30\% para desarrollar TNE Tipo 2, mientras que los pacientes con SZE esporádico, sólo tienen un riesgo de $0 \%-1 \%{ }^{9}$. Al igual que en el Tipo 1, estos tumores derivan de células ECL estimuladas por la secreción aumentada de gastrina. Hipergastrinemia producida por gastrinomas en el páncreas o duodeno. Prácticamente todos los casos muestran tumores múltiples de presentación más florida que en tumores asociados a gastritis atrófica. Se encuentran metástasis en aproximadamente $10 \%$ de los casos ${ }^{9}$.

\section{Tipo 3}

También llamado de presentación esporádica. Representa el 20\% de los TNE gástricos. No están asociados a hipergastrinemia. Pueden contener una variedad de células endocrinas y presentarse como síndrome carcinoide atípico. Morfológicamente son en general tumores únicos con un tamaño medio de $2 \mathrm{~cm}^{7}$. Tienen comportamiento más agresivo, describiéndose metástasis en $65 \%$ de los pacientes, predominantemente hepáticas. La mortalidad relacionada a carcinoide es de aproximadamente $1 / 3$ y tienen mediana de sobrevida de aproximadamente 4 años ${ }^{10}$.

\section{Tipo 4}

Tumores compuestos de otras células endocrinas como las secretores de serotonina, gastrina o ACTH, carcinomas endocrinos pobremente diferenciados y tumores mixtos endocrinos-exocrinos. A diferencia de los tumores de células ECL, pueden ubicarse en cualquier parte del estómago. Clínicamente son similares al Tipo 3, generalmente tumores únicos con mayor potencial agresivo ${ }^{8}$.

\section{Imágenes}

La mayoría de los TNE gástricos son vistos en endoscopía digestiva alta (EDA), para tumores de mayor tamaño, puede ser utilizada la endosonografía. La tomografía computada (TC) o resonancia magnética (RM) son recomendadas para evaluar la presencia de metástasis en pacientes con TNE gástricos Tipo 1 o 2 de más de $2 \mathrm{~cm}$ de diámetro o para pacientes Tipo 3. La RM es de elección en pacientes con alergia al medio de contraste ${ }^{12}$. Imágenes funcionales, como el cintigrama de receptores de somatostatina o PET 68Ga-DOTATATE, presentan mayor sensibilidad para la detección de tumor primario y metástasis, pudiendo ser utilizados de forma complementaria $^{12}$.

\section{Laboratorio}

Los exámenes de laboratorio son muy importantes, especialmente en pacientes con TNE gástrico tipo 1 y 2. En estos pacientes, se debe medir, niveles de gastrina dado que están siempre elevados.

Asimismo niveles de cromogranina $\mathrm{A}(\mathrm{CgA})$ que sirven también para seguimiento. Exámenes complementarios deben realizarse según el contexto clínico. En el caso de TNE Tipo 3, los niveles de CgA tienen su utilidad en el seguimiento ${ }^{13}$.

\section{Tratamiento}

El tratamiento es predominantemente quirúrgico, y depende del tipo de TNE gástrico. En pacientes Tipo 1 o 2, las recomendaciones varían, pero es generalmente aceptada la vigilancia anual en tumores $<10 \mathrm{~mm}$. En tumores entre $10-20 \mathrm{~mm}$, y hasta 6 pólipos, se recomienda la resección endoscópica ${ }^{9,12,13}$.

Pacientes con tumores de mayor tamaño, dado el riesgo de adenocarcinoma asociado, se recomienda una resolución más agresiva, como la resección quirúrgica local ${ }^{14}$.

En tumores Tipo 1, puede plantearse una antrectomía, con el fin de detener la estimulación de gastrina en las células ECL que a su vez genera estos TNE. Este procedimiento es efectivo en $80 \%$ de los pacientes con TNE Tipo $1^{14}$.

En el caso de progresión a tumores malignos, se recomienda la resolución quirúrgica con linfadenectomía.

Para tumores tipo 3, se propone la resección quirúrgica asociada a linfadenectomía ${ }^{14}$. Algunos autores, proponen realizar resección local a pacientes con bajo riesgo $(<8 \%)$ de tener metástasis linfáticas. Tumores menores de $2 \mathrm{~cm}$, intraepiteliales y entre 1 y $2 \mathrm{~cm}$ que invaden lámina propia o submucosa, representan el grupo de bajo riesgo y pueden ser tratados con resección endoscópica ${ }^{14}$. Otros, proponen otros criterios de bajo riesgo para realización de resección en cuña o endoscópica. Estos son, tumores bien diferenciados grado 1 , menores a $1,5 \mathrm{~cm}$ y $\sin$ invasión linfovascular ${ }^{15}$.

Sin embargo, ninguno de estos 2 enfoques representa la norma, y se sigue recomendando tratar este tipo de tumores, igual que los adenocarcinomas gástricos ${ }^{9,14}$.

Con respecto al tratamiento médico, en TNE Tipo 1 su utilidad es debatible, y su evidencia se remite a datos retrospectivos y reportes de casos. El uso de medidas antigastrina como la acidificación gástrica o los análogos de somatostatina, pueden disminuir la gastrina y la cromogranina $\mathrm{A}^{19}$. Sin embargo las medidas de vigilancia previamente descritas, siguen siendo necesarias, sin cambiar el manejo propuesto. Por esto, solamente se plantea el tratamiento médico, en pacientes que no son candidatos a terapia local, endoscópica o quirúrgica. 
En TNE tipo 2, con síndrome de Zollinger - Ellison asociado a NEM 1, se ha descrito regresión de las lesiones con el uso de análogos de somatostatina, además de la disminución en los niveles de gastrina plasmática. También podría recomendarse esta terapia, en pacientes que no son candidatos a tratamiento local.

El beneficio observado en estos 2 tipos, se debe principalmente al efecto de los análogos de somatostatina sobre la secreción de gastrina que estimula la proliferación de las células $\mathrm{ECL}^{8,10}$. Sin embargo, no se puede descartar un efecto directo estos fármacos en las células ECL. Estas células, presentan receptores específicos de somatostatina (Subtipo 2).

Es por este efecto, que se ha reportado regresión en TNE Tipo 3, posterior al uso prolongado de análogos de somatostatina, a pesar de tener niveles de gastrina normales. A pesar de esto, la recomendación sigue siendo el tratamiento quirúrgico de estos tumores.

Nuestro caso clínico, representa un hallazgo muy infrecuente dentro del diagnóstico diferencial en la sospecha de cáncer gástrico. Es por esto, que las decisiones tanto diagnósticas, como terapéuticas, deben ser tomadas de forma multidisciplinaria.

El progresivo uso de endoscopía digestiva alta, hará cada vez más frecuente el hallazgo de TNE en todos sus tipos.

Los TNE gástricos son usualmente indolentes y no funcionantes. Por esto, en nuestra paciente, sus síntomas no fueron atribuidos a su TNE.

Nuevas tecnologías como el PET 68Ga-DOTATATE, si bien tienen un mejor rendimiento diagnóstico, no se encuentran disponibles en nuestro sistema público de salud, reflejando un déficit en las herramientas diagnósticas utilizadas para esta patología.

El tratamiento de los TNE gástricos es preferentemente local, pero varía significativamente según el tipo diagnosticado.

Es por esto, que el conocimiento de esta patología, asociado a la correcta clasificación de los pacientes, es fundamental para un diagnóstico preciso que lleve al tratamiento adecuado. Las alternativas van desde el seguimiento, hasta la gastrectomía con linfadenectomía.

En este caso, la discusión multidisciplinaria, asociado a la revisión de literatura, concluyó que la mejor alternativa terapéutica, era la vigilancia endoscópica, por sobre tratamientos de mayor complejidad, como son la cirugía o los análogos de somatostatina.

La paciente expresó su preferencia por la vigilancia endoscópica. Al estar asintomática y una vez descartado el cáncer gástrico, refiere que la cirugía no sería de mayor utilidad a las medidas ya tomadas.

\section{Responsabilidades éticas}

Protección de personas y animales. Los autores declaran que para esta investigación no se han realizado experimentos en seres humanos ni en animales.

Confidencialidad de los datos. Los autores declaran que en este artículo no aparecen datos de pacientes.

Conflictos de interés: no hay.

\section{Bibliografía}

1. DeVita, Vincent T Jr. Carcinoid Tumors and Carcinoid Syndrome en: Cancer: Principles and Practice of Oncology, 10th Edition. Philadelfia: Lippincott Williams \&Wilkins, 2011, p. 8518-68.

2. Modlin I, Lye K, Kidd M. A 50-year analysis of 562 gastric carcinoids: small tumor or larger problem? Am J Gastroenterol. 2004;99:23-32. PMID: 14687136.

3. Modlin IM, Kidd M, Latich I, Zikusoka MN, Shapiro MD. Current status of gastrointestinal carcinoids. Gastroenterology 2005;128:1717-51. PMID: 15887161.

4. Obendorfer S, Frankfurt Z. Karzinoide tumoren des duenndarms. Pathol. 1907;1:426-30.

5. Askanazy M. Zur Pathogenese der Magen-krebse und uber ihren gegentlichen Ursprung aus angeboren epithelialen Keimen in der Magenwand. Dtsch Med Wochenschr. 1923;49:49-51.

6. Bosman F, Carneiro F, Hruban R, Theise N. Tumours of the stomach en: WHO Classification of Tumours of the Digestive System. 4th Edition. Lyon: International Agency for Research on Cancer 2010, p.37-66.

7. Bordi C. Endocrine tumours of the stomach. Path Res Pract. 1995;191:37380. PMID: 7479354 DOI: 10.1016/S03440338(11)80891-5.

8. Borch K, Ahrén B, Ahlman H,
Falkmer S, Granérus G, Grimelius L. Gastric Carcinoids Biologic Behavior and Prognosis After Differentiated Treatment in Relation to Type. Ann Surg. 2005;242:64-73. PMID: 15973103.

9. Delle Fave G, Capurso G, Milione M, Panzuto F. Endocrine tumours of the stomach. Best Pract Res Clin Gastroenterol. 2005;19:659-73. PMID: 16253892 DOI: 10.1016/j. bpg.2005.05.002.

10. Rindi G, Luinetti O, Cornaggia M, Capella C, Solcia E. Three subtypes of gastric argyrophil carcinoid and the gastric neuroendocrine carcinoma: a clinicopathologic study. Gastroenterology 1993;104:994-1006. PMID: 7681798. 
11. Soga J. Early-stage carcinoids of the gastrointestinal tract: an analysis of 1914 reported cases. Cancer 2005;103:158795. PMID: 15742328 DOI: 10.1002/ cncr.20939.

12. Al-Nahhas A, Win Z, Szyszko T, Singh A, Khan S, Rubello D. What can gallium-68 PET add to receptor and molecular imaging? Eur J Nucl Med Mol Imaging 2007;34:1897-901. DOI 10.1007/ s00259-007-0568-1.

13. Ruszniewski P, Delle Fave G,

Cadiot G, Komminoth P, Chung D,

Kos-Kudla B, et al. Well-

Differentiated Gastric Tumors/

Carcinomas. ENETS Guidelines.

Neuroendocrinology 2006;84:158-64.

DOI: $10.1159 / 000098007$.

14. Gladdy R, Strong V, Coit D, Allen P, Gerdes H, Shia J, et al. Defining surgical indications for type I gastric carcinoid tumor. Ann Surg Oncol. 2009;16:3154-60. PMID: 19727959 DOI: 10.1245/s10434009-0687-y.

15. Hirschowitz B, Griffith J, Pellegrin D, Cummings O. Rapid Regression of Enterochromaffinlike Cell Gastric Carcinoids in Pernicious Anemia After Antrectomy. Gastroenterology 1992;102:1409-18. PMID: 1551550. 